\title{
Auroral Energy Input From Energetic Electrons and Joule Heating at Chatanika
}

\author{
Vincent B. Wickwar and Murray J. Baron \\ Stanford Research Institute, Menlo Park, California 94025 \\ Robert D. Sears \\ Lockheed Research Laboratory, Palo Alto, California 94304
}

\begin{abstract}
With the incoherent scatter radar at Chatanika, Alaska, a wide variety of measurements can be made related to the ionosphere, magnetosphere, and neutral atmosphere. A significant parameter is the amount of energy transferred from the magnetosphere into the ionosphere and neutral atmosphere during periods of auroral activity. In this report we examine a procedure whereby the incident energy flux of auroral electrons is ascertained from radar measurements. As part of the process we compare radar-determined fluxes with those ascertained from simultaneous photometric observations at $4278 \AA$. The fluxes obtained by both techniques had similar magnitudes and time variations. If we assume that the largest uncertainty in the radar/photometer comparison is the effective recombination coefficient, then that coefficient can also be deduced. We find a value $3 \times 10^{-7} \mathrm{~cm}^{3} / \mathrm{s}$ at about $105 \mathrm{~km}$, which is in good agreement with other recent determinations during active auroral conditions. We then combine this technique with one to ascertain the Joule heating to determine the energy input from the magnetosphere to the ionosphere in a region localized above the radar on March 22, 1973, in the midnight sector. The energy input is continuous at a significant level, i.e., greater than the $3 \mathrm{ergs} / \mathrm{cm}^{2}$ s that could be delivered by the sun, were it overhead. Moreover, at times, each of these inputs became as great as $30 \mathrm{ergs} / \mathrm{cm}^{2} \mathrm{~s}$.
\end{abstract}

\section{INTRODUCTION}

In the past, measurements of particle energy input during auroral activity have been limited to brief periods when data were obtained from rockets or satellites or to periods of darkness and clear skies when data were obtained photometrically. Furthermore, such measurements were usually unaccompanied by extensive diagnostics of the state of the magnetosphere, ionosphere, and neutral atmosphere. In this report we combine incoherent scatter radar measurements from Chatanika, Alaska [Leadabrand et al., 1972], with 4278$\AA$ photometer measurements [Sears, 1973] to examine a procedure whereby the energy input from energetic electrons can be ascertained by the radar alone. The ability to determine this energy input as well as that from Joule heating [Brekke et al., 1974], when they are combined with the extensive magnetosphere and ionospheric diagnostics available from the radar [Wickwar, 1975] for extended time periods, should lead to significant data that can be applied to a wide range of problems. Examples of such problems are auroral energetics, magnetospheric particle populations, thermal structure of the ionosphere, optical emissions and compositions, thermospheric winds, and perhaps the magnitude of the solar EUV input.

In working out the steps in the radar procedure we examine the effects of electron-to-ion temperature ratio $T_{r}$, Debye length, effective recombination coefficient $\alpha_{\text {eff }}$, and altitude limitations. If the greatest uncertainty in the electron energy fluxes determined simultaneously from the radar and photometer measurements is ascribed to $\alpha_{\text {eff }}$, then we can determine that coefficient by a least squares normalization of the two fluxes.

In what follows we review, for comparison purposes, how the auroral electron energy input is ascertained from photometric observations. We then present the steps for two radar procedures. The photometric and radar procedures are

Copyright (c) 1975 by the American Geophysical Union. applied to data taken simultaneously on three nights. Finally, for March 22, 1973, the energy input to the ionosphere near Chatanika during the midnight sector is determined for both auroral electrons and Joule heating.

\section{Photometer Procedure}

The photometric observations of the $\mathrm{N}_{2}{ }^{+}(0-1) 1 \mathrm{NG}$ (first negative) band (band head at $4278 \AA$ ) are interpreted in terms of incident electron energy flux as described by Omholt [1971]. The procedure is based on similar shapes for the ionization and ionization-plus-excitation cross sections combined with the loss of $35 \mathrm{eV}$ from the electron beam for each ion pair created. For the total efficiency we used $213 \mathrm{R}$ of $\mathrm{N}_{2}^{+}(0-1)$ emission for each erg $/ \mathrm{cm}^{2} \mathrm{~s}$ of incident auroral electrons [Bortner and Bauer, 1972; Rees and Luckey, 1974].

In order to obtain the emitted band intensity the observed intensities are corrected by a factor of 1.7 for atmospheric extinction [Valley, 1965] and for the filter transmittance function, which is applied to the band shape (R. Gattinger, private communication, 1973). Absolute calibration was determined by repeated use of a radioactive phosphor source, the calibration of which is traceable to the National Bureau of Standards (M. Gadsden, private communication, 1970). The resultant uncertainty is mostly from the absolute calibration (about $30 \%$ ) and the extinction coefficient. We estimate the overall uncertainty to be less than about $50 \%$.

\section{Radar Procedures}

Whereas the photometric procedure is based upon determination of the $\mathrm{N}_{2}+(1 \mathrm{NG})$ intensity and its conversion into the column-integrated ion production rate, the radar procedures are based on determination of the electron density profile and its conversion into the ion production rate profile. The latter profile may then be integrated along the line of sight and treated identically to the photometric result, or the $35-\mathrm{eV}$ loss per ionization may be applied to the ionization rate profile to obtain the profile of the rate of energy deposition. 
The radar system is used to measure the total power backscattered from the electrons in the ionosphere. From the received power we determine a raw electron density profile [Baron et al., 1970], which has to be corrected for $T_{r}$ and Debye length effects [Baron et al., 1970; Wickwar, 1974]. Although theoretical calculations of electron and ion temperatures in auroral situations (see review by Schunk and Walker [1973]) would imply the possibility of large $T_{r}$ corrections above $110 \mathrm{~km}$, the two best radar/rocket comparisons of electron density (obtained in 1972 and 1974 (M. J. Baron and J. C. Ulwick, private communication, 1973, 1974)) imply that little if any correction is required below $160 \mathrm{~km}$. We have here followed the indication of the radar/rocket comparisons and assumed a temperature ratio of unity. However, we expect to be able to examine the question of electron and ion temperatures in the auroral $E$ layer with the radar in the near future.

By maintaining the assumption that $T_{r}$ is unity we are able to approximate the electron temperature by a simple model of the neutral temperature. We are thereby able to include a correction for Debye length effects. At the peak of the auroral $E$ layer, which is the most important region for the total energy flux determinations, this correction is small and noncritical. It is $10 \%$ for a small auroral $E$ layer with a peak density of $10^{5}$ $\mathrm{el} / \mathrm{cm}^{3}$ and decreases in a manner almost inversely proportional to increases in the electron density. However, in regions removed from the layer peak, which would be of interest for profiles of energy input, this correction assumes greater importance because it can become as large as 200 or $300 \%$. If the temperature ratios are eventually found to be greater than unity, then the magnitude of this correction would increase almost in proportion to the increase of the electron temperature above the model.

The ion production rate profile is obtained from the electron density profile by using the continuity equation

$$
q(h)=\alpha_{\text {etf }}(h) N_{e}^{2}(h)
$$

where $q$ is the ion production rate in ions $/ \mathrm{cm}^{3} \mathrm{~s}, \alpha_{\text {eff }}$ is the effective recombination coefficient in $\mathrm{cm}^{3} / \mathrm{s}, N_{e}$ is the electron density in $\mathrm{el} / \mathrm{cm}^{3}$, and $h$ is the altitude in $\mathrm{km}$. We neglect the differential term $d N_{e} / d t$, since the loss rate $\alpha_{\text {erf }} N_{e}{ }^{2}$ adjusts to changes in the production rate within approximately $10 \mathrm{~s}$, which is short in comparison with our integration time of 5 min. The divergence or transport term is also neglected.

The altitude range over which $q$ may be found depends on our knowledge of $\alpha_{\text {efr }}(h)$, where our simplifying assumptions concerning the differential and divergence terms are good approximations, and, of course, where $N_{e}(h)$ is significant. Furthermore, we are interested only in ionization due to energetic electrons. The resultant range extends from about 85 $\mathrm{km}$ to about $180 \mathrm{~km}$, which coincides with an ion population dominated by $\mathrm{NO}^{+}$and $\mathrm{O}_{2}{ }^{+}$. Below $85 \mathrm{~km}$ the ionization is largely due to energetic protons, and the $\alpha_{\text {eff }}$ changes drastically owing to the presence of negative ions and water vapor cluster ions. The possibility of ions being transported in and out of the radar beam becomes increasingly likely above about $140 \mathrm{~km}$, because ion motion is less impeded by collisions with neutrals and the proportion of $\mathrm{O}^{+}$ions with their smaller recombination coefficient may increase. (However, rocket measurements indicate that $\mathrm{O}^{+}$does not become significant below $180 \mathrm{~km}$ in auroral situations [Zhludko et al., 1970; Johnson, 1972; Kopp et al., 1973].)

The profile of the rate of energy deposition from auroral electrons $\epsilon(h)$ is proportional to $q(h)$, since an electron beam loses $35 \mathrm{eV}$ for the creation of each electron pair. Hence $\epsilon(h)$ 'is directly dependent on the assumptions used to derive $q(h)$ and is likely to be subject to considerable uncertainty at the end points, particularly at the upper end. However, the integrated rate of energy input, or energy flux, is largely independent of these uncertainties because most of the contribution comes from near the peak of the ionization profile, which is usually between 90 and $130 \mathrm{~km}$. Furthermore, most of the contribution to the integral comes from the immediate vicinity of the peak because of the $N_{e}^{2}$ dependence of $q$ and $\epsilon$ and the 'peakedness' of an auroral $E$ layer. In practice, on the days studied we found no contribution to the integral below $85 \mathrm{~km}$, and we found that the contributions asymptoted toward zero as the upper boundary was raised from 140 to 160 and then to $180 \mathrm{~km}$.

We shall call the above procedure for finding the energy flux the 'whole spectrum technique' because primary and secondary auroral electrons from a wide range of energies are involved. A second procedure, which we shall call the 'monoenergetic technique,' involves comparison of the maximum ionization rate and its altitude to the theoretical ionization rate curves for monoenergetic electrons of Rees [1963] or Banks et al. [1974]. The comparison enables us to find the energy flux of monoenergetic electrons that would create a production rate equal to the maximum observed. The result is a lower bound because it leaves out all the lower-energy electrons that in reality also contribute to the ionization.

\section{The DATA}

We have examined sequences of data from March 22 and April 1 and 2, 1973, when the radar and photometer were both operating. On April 1 and 2 they were coaligned looking up the magnetic field line for a total of about 6 hours. On March 22 they were nearly coaligned for about 1.5 hours out of 4 hours of simultaneous observations.

Figure 1 directly compares the energy input calculated from the photometer and from the two radar procedures for the most active day. The three measurements track each other very well (with the exception of one photometer point that might be related to its larger field of view, $3^{\circ}$ versus $0.5^{\circ}$ ). As is expected, the monoenergetic technique yields lower values than the whole spectrum technique. However, the ratios of the two radar techniques vary. We suggest that the auroral electrons

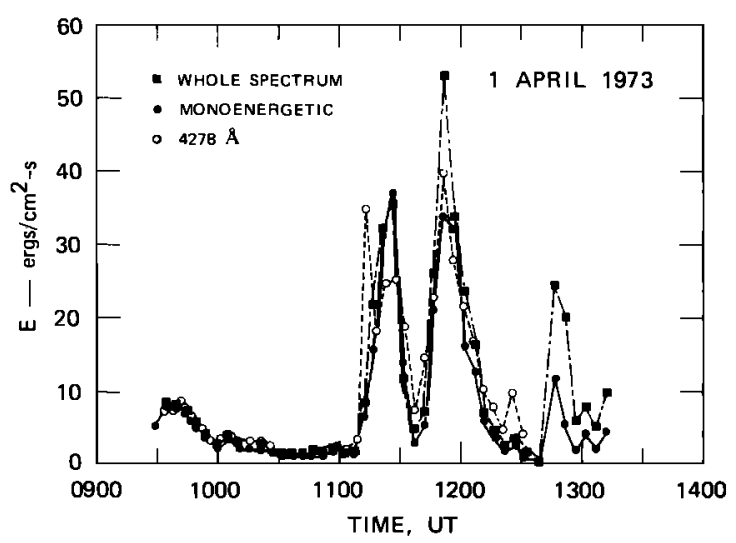

Fig. 1. Energy input from auroral electrons on April 1, 1973. It is determined by both radar procedures and the photometric procedure (see text). For all three procedures the integration time is $5 \mathrm{~min}$. Both instruments are observing up the magnetic field line. Alaska standard time is UT - 10 hours. 
are more nearly monoenergetic when the ratio approaches unity.

Figure 2 presents a direct comparison of all the simultaneous radar (whole spectrum technique) and photometer energy flux determinations along with a weighted least squares fitted line. We see good agreement between the techniques over a wide range of energy fluxes, as is expected for a linear correlation coefficient of 0.95 .

In making the comparisons it was assumed that the greatest uncertainty was in $\alpha_{\text {erf }}$. Consequently, it was treated as a parameter to be refined in optimizing the radar/photometer comparisons. Figure 3 shows the results and places them in the context of recent laboratory and experimental values. Laboratory measurements of $\mathrm{O}_{2}{ }^{+}$[Mehr and Biondi, 1969] and $\mathrm{NO}^{+}$[Biondi, 1969] recombination coefficients are combined with the neutral temperature profile for a $1000^{\circ} \mathrm{K}$ exospheric temperature [Banks and Kockharts, 1973] to produce curves A and B. Recent determinations of $\alpha_{\text {eff }}$ during active auroras with the radar [Baron, 1972, 1974] and with a rocket [Ulwick and Baron, 1973] are given in curves $C$ and $D$. Curves parallel to 1,2 , and 3 were used for full sets of calculations, and comparisons similar to the one in Figure 2 were produced. When the comparisons were optimized, curves 1,2 , and 3 were obtained. However, for the data in our sample the three fits are equally good. The relative histogram of peak altitudes for the coaligned measurements on March 22 and April 1 and 2, 1973, indicates that the indistinguishability of the curves is largely due to most of the data coming from a very limited altitude range, $100-110 \mathrm{~km}$. Hence it is not surprising that the three curves nearly intersect at $105 \mathrm{~km}$, at a value of $3 \times 10^{-7} \mathrm{~cm}^{3} / \mathrm{s}$. However, from the description of the radar procedures we recall that $N_{e}(h)$ could be systematically underestimated. If that is the case, then the derived value of $\alpha_{\text {efr }}$ would be smaller.

Because of what appears to be a more reasonable altitude dependence, curve 2 was used for the radar calculations in Figures 1, 2, and 4.

\section{COMPARISONS OF ENERGY INPUT}

Figure 4 shows energy input for both Joule heating and energetic electrons for March 22, 1973. In addition, the $H$ component of the College magnetometer is included. Increases

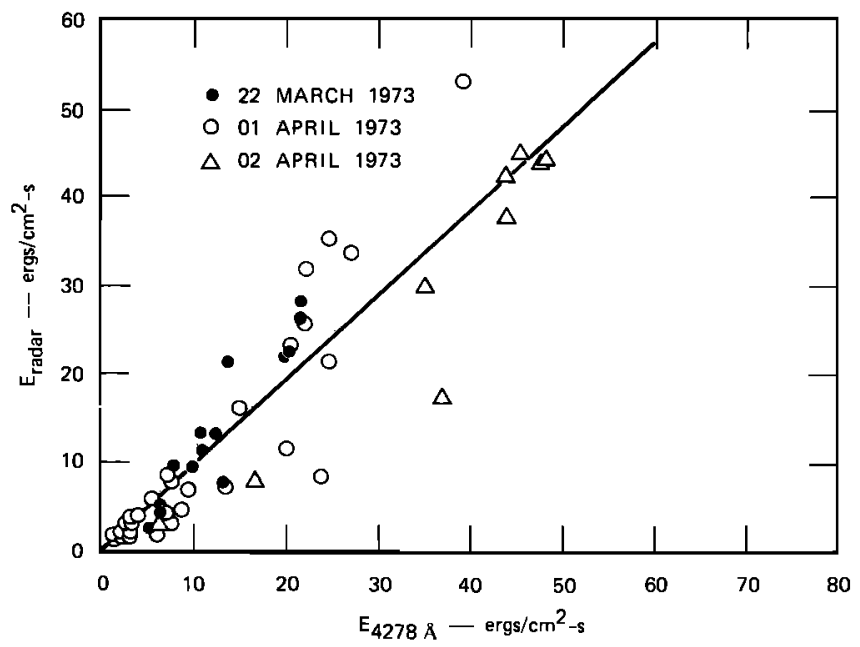

Fig. 2. Scatter plot of photometer and radar determinations of auroral electron energy input. The radar contribution uses the whole spectrum technique. All the coincident radar and photometer data (5min averages) from March 22 and April 1 and 2, 1973, are included. The line is a weighted least squares fit.

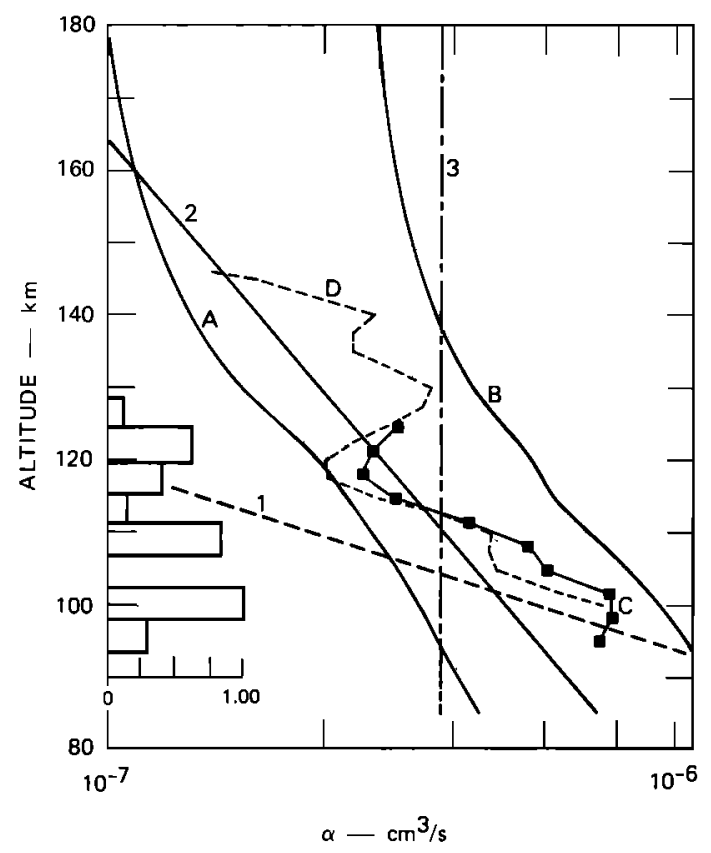

Fig. 3. Recombination coefficients and histogram of auroral $E$ layer peak altitudes (see text). Curve 2 is used for the radar results in the other figures.

in the energy input and magnetic activity generally occur together.

The curves show increases in the energy input from Joule heating and auroral electrons occurring at about the same time (within the 30 -min time resolution of the Joule heating determination). Since the energetic auroral electrons enhance the $E$ layer ionization and consequently the Pedersen conductivity, they provide a necessary condition for enhanced currents and Joule heating. Additionally, since perpendicular electric fields are a common feature of the auroral oval, a gross time correspondence between the two energy inputs is to be expected. The energy input from auroral electrons is seen to vary from about $\frac{1}{3}$ to 4 times that from Joule heating. However, since the electron input is subject to time variations of the order of a few seconds, it should be noted that the 5-min averaging (of both radar and photometer data) reduces the peak input, and the

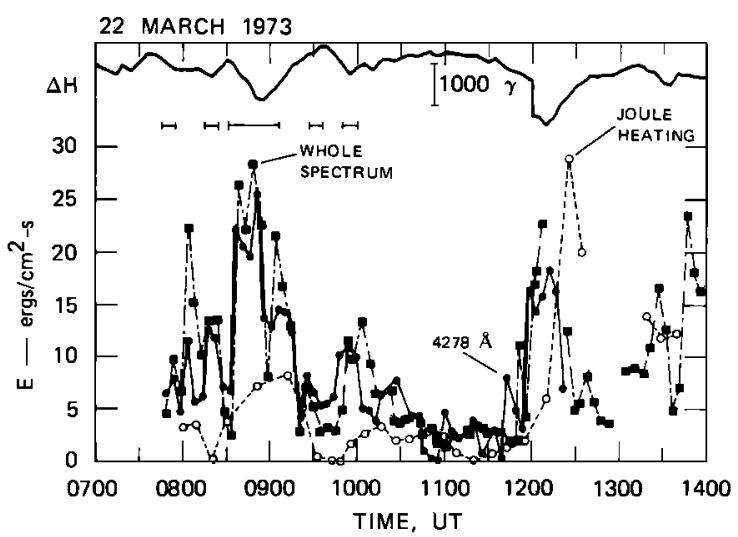

Fig. 4. Simultaneous measurements of energy input from auroral electrons and Joule heating on March 22, 1973. The electron input is determined from the radar with the whole spectrum technique and from the $4278-\AA$ photometer measurements. The Joule heating is determined with the radar. The bars indicate the periods when the radar and photometer are observing in the same direction. The $\Delta H$ component of the College magnetogram is included for reference. 
30-min smearing of Joule heating undoubtedly has a similar effect. (On other days we have seen ratios of an order of magnitude in both directions.) The base level for the combined auroral energy inputs is about $3 \mathrm{ergs} / \mathrm{cm}^{2} \mathrm{~s}$. During the two large events the input is about 1 order of magnitude larger. So far, the maximum energy input seen has been about 100 ergs $/ \mathrm{cm}^{2} \mathrm{~s}$.

An immediate consequence of the large energy inputs is that we expect large increases in the electron and ion temperatures. These data have been examined, and such increases have been seen to occur in both the electron and the ion temperatures at altitudes above $170 \mathrm{~km}$ [Baron and Chang, 1974; Wickwar, 1975].

\section{CONCLUSIONS}

In conclusion, there are four points we would like to make. First, we have combined radar and photometric observations to examine two related procedures to derive the energy input from auroral electrons by using incoherent scatter radar. The techniques are independent of seeing conditions and in principle can be extended to daytime observations. Second, we have shown that the energy inputs to the ionosphere from the magnetosphere due to Joule heating and energetic electrons can be obtained simultaneously from incoherent scatter radar measurements. Third, for extended periods of time around the midnight sector there is considerable energy input, equal to or greater than the solar energy input that would be deposited in the same part of the atmosphere. Fourth, we found a value of $\alpha_{\text {eff }}$ of $3 \times 10^{-7} \mathrm{~cm}^{3} / \mathrm{s}$ at about $105 \mathrm{~km}$, which is consistent with other recent measurements. In principle, given sufficient data from a range of altitudes, we would find a profile of $\alpha_{\text {eff }}$.

Acknowledgments. We would like to thank members of the incoherent scatter group at the Stanford Research Institute and the University of California at San Diego for helpful discussions during the preparation of this report. In particular, we would like to thank $R$. Vondrak and J. Siren. The research was in part supported by contract DNA 001-74-C-0167 from the Defense Nuclear Agency and by grants from the Atmospheric Sciences Section, National Science Foundation, to the Stanford Research Institute and by contract DNA 001-74-C0179 from the Defense Nuclear Agency to Lockheed Research Laboratory.

The Editor thanks M. H. Rees for his assistance in evaluating this report.

\section{REFERENCES}

Banks, P. M., and G. Kockharts, Aeronomy, Academic, New York, 1973.

Banks, P. M., R. W. Schunk, and W. J. Raitt, $\mathrm{NO}^{+}$and $\mathrm{O}^{+}$in the high latitude $F$-region, Geophys. Res. Lett., 1, 239-242, 1974.

Baron, M. J. (Ed.), DNA project 617 radar: Auroral ionospheric measurements, final report, SRI Proj. 1703, Stanford Res. Inst. Menlo Park, Calif., 1972.

Baron, M. J., Electron densities within aurorae and other auroral E-region characteristics, Radio Sci., 9, 341-348, 1974.

Baron, M. J., and N. J. Chang, Icecap ' $73 a-$ Chatanika radar results, Tech. Rep. 4, SRI Proj. 3118, Stanford Res. Inst., Menlo Park, Calif., 1974.

Baron, M. J., O. de la Beaujardiere, and B. Craig, Project 617 radar readiness achievement program, part A-Data processing and analysis, final report, part A, SRI Proj. 6291, Stanford Res. Inst., Menlo Park, Calif., 1970.

Biondi, M. A., Atmospheric electron-ion and ion-ion recombination processes, Can. J. Chem., 47, 1711-1719, 1969.

Bortner, M. H., and T. Baurer (Eds.), DNA Reaction Rate Handbook, 2nd ed., p. 24, DASA Inform. and Anal. Center, General Electric Tempo, Santa Barbara, Calif., 1972.

Brekke, A., J. R. Doupnik, and P. M. Banks, Incoherent scatter measurements of $E$ region conductivities and currents in the auroral zone, J. Geophys. Res., 79, 3773-3790, 1974

Johnson, C. Y., Positive ion mass spectrometer observations in the $E$ region, Radio Sci., 7, 99-101, 1972.

Kopp, E., P. Eberhardt, and J. Geiss, Ion composition in the $E$-and lower $F$-region above Kiruna during sunset and sunrise, Planet. Space Sci., 21, 227-238, 1973.

Leadabrand, R. L., M. J. Baron, J. Petriceks, and H. F. Bates, Chatanika, Alaska, auroral-zone incoherent-scatter facility, Radio Sci., 7, 747-756, 1972

Mehr, F. J., and M. A. Biondi, Electron temperature dependence of recombination of $\mathrm{O}_{2}^{+}$and $\mathrm{N}_{2}{ }^{+}$ion with electrons, Phys. Rev., 181, 264-271, 1969.

Omholt, A., The Optical Aurora, Springer, New York, 1971.

Rees, M. H., Auroral ionization and excitation by incident energetic electrons, Planet. Space Sci., 11, 1209, 1963.

Rees, M. H., and D. Luckey, Auroral electron energy derived from ratio of spectroscopic emissions, 1, Model computations, J. Geophys. Res., 79, 5181-5186, 1974.

Schunk, R. W., and J. C. G. Walker, The theory of charged particle temperatures in the upper atmosphere, in Progress in High Temperature Physics and Chemistry, edited by C. A. Rouse, pp. 1-62, Pergamon, New York, 1973.

Sears, R. D., Versatile family of modular aurora and airglow photometers, Appl. Opt., 12, 1349, 1973.

Ulwick, J. C., and M. J. Baron, Simultaneous rocket probe and incoherent-scatter measurements during an aurora, paper presented at URSI Conference on Incoherent Scatter, Troms $\$$, Norway, June 12-16, 1973.

Valley, S. L. (Ed.), Handbook of Geophysics and Space Environment, pp. 7-21, McGraw-Hill, New York, 1965.

Wickwar, V. B., Analysis techniques for incoherent-scatter data interpretation in the 100 -to- $300 \mathrm{~km}$ region, Tech. Rep. $3, S R I$ Proj. 1703, Stanford Res. Inst., Menlo Park, Calif., 1974.

Wickwar, V. B., Chatanika radar measurements, Atmospheres of Earth and the Planets, edited by B. M. McCormac, D. Reidel, Dordrecht, Netherlands, 1975.

Zhludko, A. D., G. F. Tulinov, N. M. Klyuyeva, and Yu. K. Chasovitin, Ion composition of the polar ionosphere, Geomagn. Aeron., 10, 586-588, 1970.

(Received March 24, 1975; accepted May 22, 1975.) 